\title{
Exact Expression and Tight Bound on Pairwise Error Probability for Performance Analysis of Turbo Codes over Nakagami- $m$ Fading Channels
}

\author{
Syed Amjad Ali, Member, IEEE, Nirmal Singh Kambo, and Erhan AliRiza İnce, Member, IEEE
}

\begin{abstract}
This letter presents derivation for an exact and efficient expression on pairwise error probability over fully interleaved Nakagami- $m$ fading channels under ideal channel state information at the decoder. As an outcome, this derivation also leads to a tight upper bound on pairwise error probability which is close to the exact expression. Pairwise error probability plots for different values of Nakagami parameter $m$ along with an already existing numerically computable expression are provided. As an application of pairwise error probability, average union upper bounds for turbo codes having (1,7/5,7/5) and $(1,5 / 7,5 / 7)$ generator polynomials employing transfer function approach are presented to illustrate the usefulness of the new efficient results.
\end{abstract}

Index Terms-Average union upper bound, ideal channel state information, Nakagami- $m$ fading channel, pairwise error probability, turbo codes.

\section{INTRODUCTION}

$\mathbf{T}$ HE impressive performance of turbo codes over both the additive white Gaussian noise (AWGN) and fading channels is thoroughly discussed in [1]-[4]. Bit error probability bounds are mostly used for the performance analysis of turbo codes for large values of signal to noise ratio $\left(R E_{b} / N_{0}\right)$ to avoid extensive simulation time. On the contrary, both simulation and tight upper bounds beyond the channel cutoff rate can be used to study the code performance. Unfortunately, tight upper bounds exist only for the AWGN and Rician fading channel case with the expressions provided by Sason [5] being the prominent ones.

Recently, authors in [6], [7] provided probability distribution based derivation for pairwise error probability over Rayleigh and Rician fading channels and showed that this approach led to computationally efficient results. This letter follows a similar approach to derive an exact and efficient expression for pairwise error probability over fully interleaved Nakagami- $m$ fading channels for BPSK modulation. The newly derived expression is also approximated to yield a tight upper bound which stays close to the exact expression. Using these new results, union upper bounds are obtained which are computationally more efficient than an existing exact pairwise error probability expression which is based on numerical integration. A rate $1 / 3$ turbo code with a memory of

Manuscript received January 16, 2007. The associate editor coordinating the review of this letter and approving it for publication was Dr. Giorgio Taricco.

S. A. Ali is with the Department of Computer Technology and Information Systems, Bilkent University, Ankara, Turkey (e-mail: syedali@bilkent.edu.tr).

N. S. Kambo is Ex. Prof. IIT, Delhi, India and EMU, Famagusta, North Cyprus (e-mail: nskambo@yahoo.com).

E. A. Ince is with the Department of Electrical and Electronic Engineering, Eastern Mediterranean University, Famagusta, North Cyprus, via Mersin 10, Turkey (e-mail: erhan.ince@emu.edu.tr).

Digital Object Identifier 10.1109/LCOMM.2007.070082. two and an input block size of $K$ bits and an output encoded stream of $N=3(K+2)$ bits is used to study the pairwise error probability expressions. With both encoders terminating in the zero state, union upper bounds for $(1,7 / 5,7 / 5)$ and $(1,5 / 7,5 / 7)$ code structures are obtained to demonstrate the application of the proposed results.

The remaining sections of this letter are organized in the following order. Section II discusses details regarding transfer function based average union upper bounds. Section III presents an existing expression for pairwise error probability together with the newly derived results. In Section IV results are discussed. Lastly, Section V summarizes the findings of the work.

\section{Transfer Function BASEd Union Bounds}

The rate $1 / 3$ turbo code is a parallel concatenated error correction coding scheme. The encoder output is transmitted as the multiplexed version of the message sequence with Hamming weight $i$ (the systematic bit) together with the two parity sequences with Hamming weights of $d_{1}$ and $d_{2}$. The parity sequences are obtained by encoding the message sequence and the interleaved version of the message. Since the codeword is obtained by concatenating the three sequences, the Hamming weight $d$ of the codeword becomes the sum of the individual weights of these three sequences (i.e., $d=$ $\left.i+d_{1}+d_{2}\right)$. Following [2] and [4], union upper bound on the ensemble average value of bit error probability can be written as

$$
P_{b} \leq \sum_{i=1}^{K} \sum_{d_{1}} \sum_{d_{2}} \frac{i}{K}\left(\begin{array}{c}
K \\
i
\end{array}\right) p\left(d_{1} \mid i\right) p\left(d_{2} \mid i\right) P_{2}(d)
$$

where $p(d \mid i)$ is the conditional probability of producing a codeword fragment of weight $d$ for a randomly selected input sequence of weight $i, P_{2}(d)$ represents the pairwise error probability and the Hamming weight $d$ of the code starts from $d_{\text {min }}=\left(i+d_{1}+d_{2}\right)_{\text {min }}$ which is based on the selected code [3]. In order to evaluate the expression in (1) the distribution of the weight of parity sequences needs to be determined. The expression for their distribution is attained as shown by [2] and equals

$$
p(d \mid i)=\frac{t(l, i, d)}{\left(\begin{array}{c}
K \\
i
\end{array}\right)}
$$

where $t(l, i, d)$ is obtained from the code's transfer function and represents the total number of paths of length $l$, input weight $i$, and output weight $d$, emerging from and terminating in the zero state. 


\section{PAIRWISE ERror Probability}

For a fading channel with ideal channel state information, the conditional pairwise error probability of decoding a codeword $\mathbf{c}_{\mathbf{o}}$ into a codeword $\mathbf{c}_{\mathbf{j}}$ which differs from $\mathbf{c}_{\mathbf{o}}$ in $d$ bit positions indexed by $(1,2, \ldots, d)[4],[8]$ is

$$
P\left(\mathbf{c}_{\mathbf{o}}, \mathbf{c}_{\mathbf{j}} \mid \mathbf{a}\right)=Q\left[\sqrt{\frac{2 R E_{b}}{N_{0}} \sum_{k=1}^{d} a_{k}^{2}}\right]
$$

where $Q(x)$ is the Gaussian Q-function given by

$$
Q(x)=\frac{1}{\sqrt{2 \pi}} \int_{x}^{\infty} e^{-t^{2} / 2} d t .
$$

The pairwise error probability, $P_{2}(d)$, is found by computing the expected value of the conditional probability in (3). In what follows, we list the only known exact expression for $P_{2}(d)$ along with the new results that the authors have derived.

\section{A. Exact 1}

An exact numerically computable finite integral based expression for pairwise error probability is provided in [9] as

$$
P_{2}(d)=\frac{1}{\pi} \int_{0}^{\frac{\pi}{2}}\left(\frac{m \sin ^{2} \theta}{R E_{b} / N_{0}+m \sin ^{2} \theta}\right)^{m d} d \theta
$$

where $m$ is the Nakagami fading parameter. Though, the above integral can be computed numerically it may require more computational time for fairly large values of $m d$ and $E_{b} / N_{0}$.

\section{B. Exact 2}

This new pairwise error probability expression is derived in Appendix I and has the following infinite series representation

$$
P_{2}(d)=\frac{\Delta^{m d}}{2 \sqrt{\pi}} \sqrt{\frac{R E_{b} / N_{0}}{m+R E_{b} / N_{0}}} \sum_{k=0}^{\infty} \frac{\Gamma\left(m d+k+\frac{1}{2}\right) \Delta^{k}}{\Gamma(m d+k+1)}
$$

where

$$
\Delta=\frac{m}{m+R E_{b} / N_{0}} .
$$

Even though, the above series is infinite it is sufficient to evaluate the first 10 terms for values of $m \leq 1$ and first 30 terms for $m>1$ to achieve reasonable accuracy.

\section{Bound}

This new bound is obtained by bounding the infinite series given in (6) (see Appendix I)

$$
P_{2}(d) \leq \frac{\Gamma\left(m d+\frac{1}{2}\right) \Delta^{m d}}{2 \sqrt{\pi} \Gamma(m d+1)} \sqrt{\frac{m+R E_{b} / N_{0}}{R E_{b} / N_{0}}} .
$$

The bound matches perfectly with the exact expression (6) for most of the values of $E_{b} / N_{0}$ as shown in Fig. 1. Minor difference between the exact expression and bound exist for small values of $m$ and $E_{b} / N_{0}$.

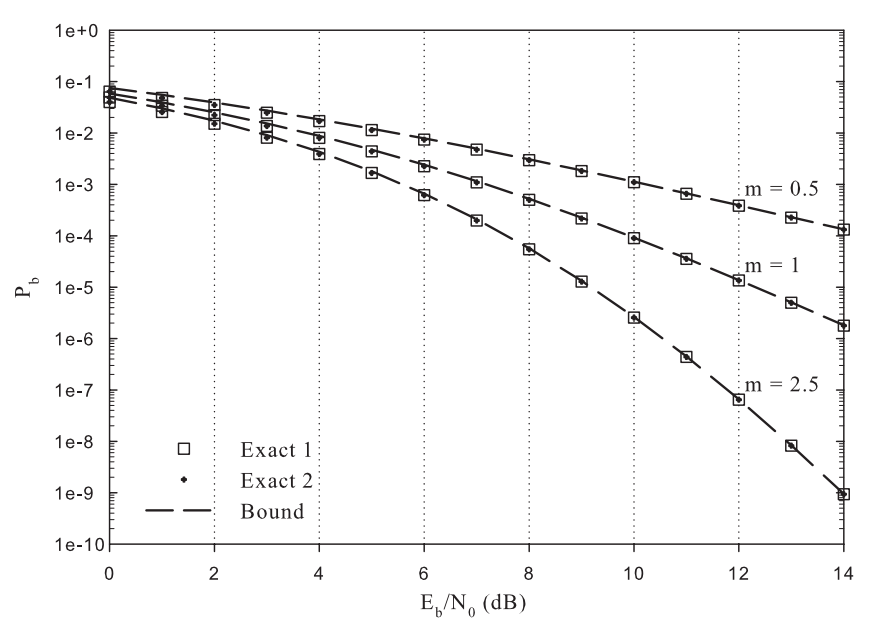

Fig. 1. Pairwise error probability plots for $d=5$ over fully interleaved Nakagami- $m$ fading channels $(m=0.5,1,2.5)$.

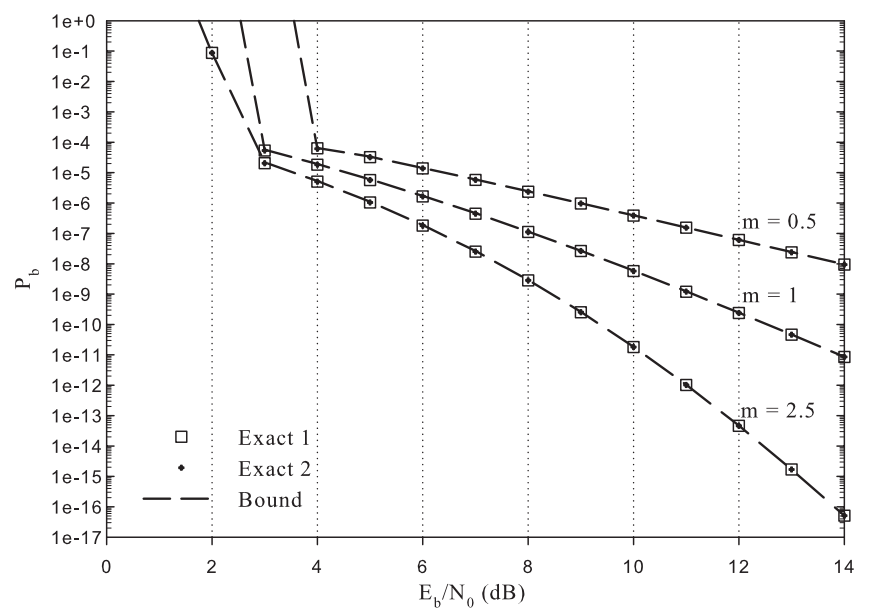

Fig. 2. Bit error probability bounds for $(1,7 / 5,7 / 5)$ turbo code over fully interleaved Nakagami- $m$ fading channels $(m=0.5,1,2.5)$ using message block length of $K=1000$.

\section{RESUlts AND Discussion}

Fig. 1 shows the plots for the exact value and upper bounds on the pairwise error probability for $d=5$ and $m$ having values $0.5,1$ and 2.5 to encompass the cases of worst case fading scenario of a one-sided Gaussian distribution, Rayleigh and mild fading respectively. The plots clearly depict that the derived Bound is in close approximation to the exact result and hence can be used to obtain similar results as those by exact expression with a considerable reduction in computational complexity. Fig. 2 and Fig. 3 show the upper bounds on average bit error probability for two selected codes with message block length of 1000 bits. The union bounds obtained by using expression (6) and the bound (8) for $P_{2}(d)$ in (1) are found to be almost identical. Additionally, the average bit error probability bounds for $(1,5 / 7,5 / 7)$ code are slightly better than those of $(1,7 / 5,7 / 5)$ due to the better distance properties of the prior code [3]. 


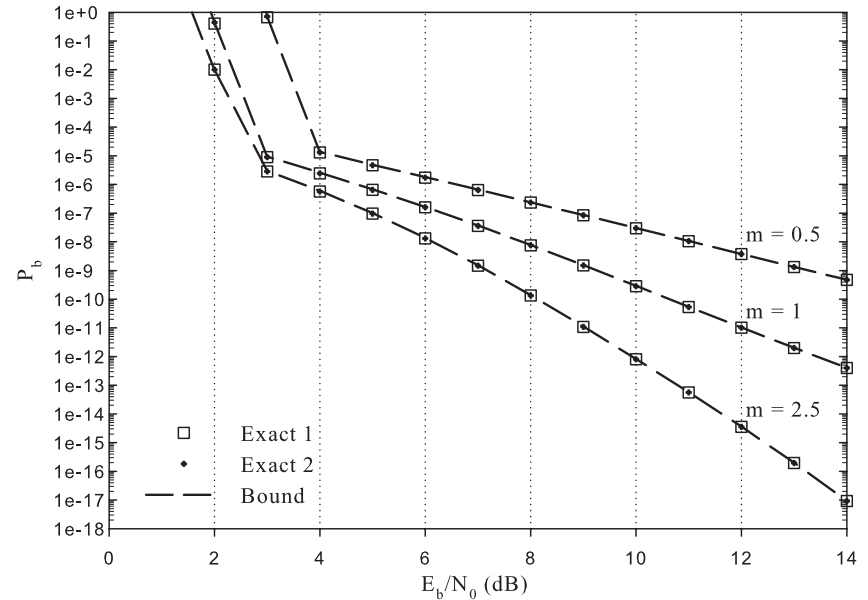

Fig. 3. Bit error probability bounds for $(1,5 / 7,5 / 7)$ turbo code over fully interleaved Nakagami- $m$ fading channels $(\mathrm{m}=0.5,1,2.5)$ using message block length of $K=1000$.

\section{CONCLUSION AND REMARKS}

This letter gives the derivation of a new exact expression for the pairwise error probability over independent Nakagami- $m$ fading channels with ideal channel state information at the decoder. The derivation also leads to a tight upper bound which follows the exact result closely. More importantly, the use of this concise and efficient pairwise error probability expression has considerably reduced the computation time of the average union upper bounds.

\section{APPENDIX I}

The normalized probability density function $\left(E\left[a^{2}\right]=1\right)$ for the Nakagami- $m$ distribution [10] can be written as

$$
p(a)=\frac{2 m^{m} a^{2 m-1} e^{-m a^{2}}}{\Gamma(m)}, \quad m \geq \frac{1}{2}, \quad a \geq 0
$$

where $m$ denotes the fading parameter and $\Gamma(\cdot)$ is the gamma function. The pairwise error probability is defined as the expected value of (3) and can be expressed as

$$
P_{2}(d)=E\left\{Q\left(\sqrt{\frac{2 R E_{b}}{N_{0}} Z}\right)\right\}
$$

where $Z=\sum_{k=1}^{d} a_{k}^{2}$ and $\left(a_{1}, a_{2}, \ldots, a_{d}\right)$ are i.i.d. Nakagami$m$ random variables. Since $a_{k}^{2}$ has Gamma pdf $f(x)=$ $\frac{m^{m}}{\Gamma(m)} x^{d-1} e^{-m x}, x \geq 0, Z$ also has Gamma pdf [11] given as

$$
p_{Z}(z)=\frac{m^{m d}}{\Gamma(m d)} z^{m d-1} e^{-m z}, \quad z \geq 0 .
$$

Thus, from (10) and (11) we get

$$
P_{2}(d)=\int_{0}^{\infty}\left(\frac{m^{m d}}{\sqrt{2 \pi}} \int_{\sqrt{\frac{2 R E_{b}}{N_{0}} z}}^{\infty} e^{-\frac{t^{2}}{2}} d t\right) \frac{z^{m d-1} e^{-m z}}{\Gamma(m d)} d z .
$$

Changing the order of integration, (12) becomes

$$
P_{2}(d)=\int_{0}^{\infty} \frac{e^{-t^{2} / 2}}{\sqrt{2 \pi} \Gamma(m d)}\left(\int_{0}^{\frac{m}{2 R E_{b} / N_{0}} t^{2}} x^{m d-1} e^{-x} d x\right) d t .
$$

The inner integral in (13) is the lower incomplete Gamma function which has an infinite series representation [12] of

$$
\int_{0}^{u} x^{p-1} e^{-x} d x=e^{-u} \sum_{k=0}^{k=\infty} \frac{\Gamma(p) u^{p+k}}{\Gamma(p+k+1)} .
$$

Substituting (14) into (13), simple calculations yield

$$
P_{2}(d)=\frac{\Delta^{m d}}{2 \sqrt{\pi}} \sqrt{\frac{R E_{b} / N_{0}}{m+R E_{b} / N_{0}}} \sum_{k=0}^{\infty} \frac{\Gamma\left(m d+k+\frac{1}{2}\right) \Delta^{k}}{\Gamma(m d+k+1)}
$$

where $\Delta$ was defined previously in (7).

\section{Upper bound on pairwise error probability}

The term $\frac{\Gamma\left(m d+k+\frac{1}{2}\right)}{\Gamma(m d+k+1)}$ is monotonically decreasing with $k$, it follows from (15) that

$$
P_{2}(d) \leq \frac{\Gamma\left(m d+\frac{1}{2}\right) \Delta^{m d}}{2 \sqrt{\pi} \Gamma(m d+1)} \sqrt{\frac{m+R E_{b} / N_{0}}{R E_{b} / N_{0}}} .
$$

\section{REFERENCES}

[1] C. Berrou, A. Glavieux, and P. Thitimajshima, "Near Shannon limit errorcorrecting coding and decoding: turbo codes," in Proc. IEEE Int. Conf. on Communications (ICC), 1993, pp. 1064-1070.

[2] D. Divsalar, S. Dolinar, R. McEliece, and F. Pollar, "Transfer function bounds on the performance of turbo codes," JPL TDA Progress Report, vol. 42, Aug. 1995, pp. 44-55.

[3] S. Benedetto and G. Montorsi, "Unveiling turbo codes: some results on parallel concatenated coding schemes," IEEE Trans. Inf. Theory, vol. 42, pp. 409-429, Mar. 1996.

[4] E. K. Hall and S. Wilson, "Design and analysis of turbo codes on Rayleigh fading channels," IEEE J. Sel. Areas Commun., vol. 16, no. 2, pp. 160-174, Feb. 1998.

[5] I. Sason and S. Shamai (Shitz), "On improved bounds on the decoding error probability of block codes over interleaved fading channels, with applications to turbo-like codes," IEEE Trans. Inf. Theory, vol. 47, no. 6, pp. 2275-2299, Sept. 2001.

[6] E. A. İnce, N. S. Kambo, and S. A. Ali, "Efficient expression and bound for pairwise error probability in Rayleigh fading channels, with application to union bounds for turbo codes," IEEE Commun. Lett., vol. 9, no. 1, pp. 25-27, Jan. 2005.

[7] S. A. Ali and E. A. İnce, "Closed form expression and improved bound on pairwise error probability for performance analysis of turbo codes over Rician fading channels," IEEE Commun. Lett., vol. 10, no. 8, pp. 599-601, Aug. 2006.

[8] J. Yuan, W. Feng, and B. Vucetic, "Performance of parallel and serial concatenated codes on fading channels," IEEE Trans. Commun., vol. 50, no. 10, pp. 1600-1608, Oct. 2002.

[9] M. K. Simon and M-S. Alouini, "A unified approach to the performance analysis of digital communication over generalized fading channels," Proc. of the IEEE, vol. 86, no. 9, pp. 1860-1877, Sept. 1998.

[10] M. Nakagami, "The $m$-distribution, a general formula of intensity distribution of rapid fading," Statistical Methods in Radio Wave Propagation, W. G. Hoffman, Ed. Oxford, England: Pergamon, 1960.

[11] A. Papoulis and S. U. Pillai, Probability, Random Variables and Stochastic Processes, Fourth Edition. McGraw Hill, 2002.

[12] I. S. Gradshteyn and I. M. Ryzhik, Tables of Integrals, Series and Products, Sixth Edition. San Diego: Academic Press, 2000. 